\title{
A set of exactly solvable Ising models with half-odd-integer spin
}

\author{
Onofre Rojas*and S. M. de Souza \\ Departamento de Ciências Exatas, Universidade Federal de Lavras, \\ CP 3037, 37200-000, Lavras - MG, Brazil.
}

November 28, 2018

\begin{abstract}
We present a set of exactly solvable Ising models, with half-odd-integer spin-S on a square-type lattice including a quartic interaction term in the Hamiltonian. The particular properties of the mixed lattice, associated with mixed half-odd-integer spin-(S,1/2) and only nearest-neighbor interaction, allow us to map this system either onto a purely spin-1/2 lattice or onto a purely spin-S lattice. By imposing the condition that the mixed half-odd-integer spin-(S,1/2) lattice must have an exact solution, we found a set of exact solutions that satisfy the free fermion condition of the eight vertex model. The number of solutions for a general half-odd-integer spin-S is given by $S+1 / 2$. Therefore we conclude that this transformation is equivalent to a simple spin transformation which is independent of the coordination number.
\end{abstract}

Keywords: Mathematical physics, two dimensional Ising model, exact results.

Two-dimensional lattice models are one of the most interesting subjects of statistical mechanics, both experimentally [1, 2] and theoretically. Several approximation methods are used to investigate these models on the lattice, such as mean-field theory [1, 3, the Bethe approximation [4, the correlated effective field theory [5], the renormalization group [6], series expansion methods [7, Monte Carlo methods $[$ ] and cluster variation methods. Following Onsager's [9] solution for the square two dimensional Ising lattice, other solutions for regular

${ }^{*}$ Corresponding author. email: onofre@pq.cnpq.br, Tel.: +5535 38291954, Fax.: +5535 38291371 
two-dimensional lattices have been considered, such as triangular [10, 11, honeycomb 12, 13, kagomé 14] lattices and others were explored in several works and their importance in statistical physics has waked up the search for a set of completely solvable models. Some other exact results have been obtained with restricted parameters, as investigated by Mi and Yang[16] using a non-one-to-one transformation[15].

Some half-odd-integer spin-S Ising models were already discussed in the literature[17. Using the method proposed by $\mathrm{Wu}[18$, Izmailian [19] obtained an exact solution for a spin-3/2 square lattice with only nearestneighbor and two-body spin interactions. Izmailian and Ananikian[20] have also obtained an exact solution for a honeycomb lattice with spin-3/2. A particular case of solutions of these models were obtained by the method proposed by Joseph[21, where any spin-S could be decomposed in terms of spin-1/2. Another interesting method for mapping the spin-S lattice into a spin-1/2 lattice has been proposed by Horiguchi[22].

Mapping between models is an important tool for the study of exactly solvable models. The aim of this letter is to present the transformation of higher half-odd-integer spin-S systems into a simple spin-1/2 system.

To demonstrate this transformation we use a two dimensional mixed spin-(S,1/2) on a square Ising lattice with a quartic interaction $\mathcal{L}_{b}$ as displayed in fig 1 . First this mixed model can be mapped onto an exactly solvable two dimensional spin-1/2 Ising model with a quartic interaction[26, $\mathcal{L}_{a}$, such as presented in the literature 19 . Second it is also possible to transform a mixed spin lattice $\mathcal{L}_{b}$ onto an effective spin-S lattice when we consider the spin-1/2 as a decorated Ising model of the lattice $23, \mathcal{L}_{b}$. Then the classical Hamiltonian for a mixed spin- $(3 / 2,1 / 2)$ lattice is given by

$$
\mathcal{H}_{1 / 2,3 / 2}=\sum_{<i, j>}\left(K_{r}^{(1)} S_{i} \sigma_{j}+K_{r}^{(3)} S_{i}^{3} \sigma_{j}\right)+\sum_{i} D S_{i}^{2}
$$

with $\langle i, j>$ meaning summation over nearest interacting neighbors on the square lattice, and the last summation is performed over all spin-3/2 sites. The coefficient $K_{r}^{(1)}$ is the nearest-neighbor interaction parameter of the bilinear term; $K_{r}^{(3)}$ corresponds to the parameter of the non-bilinear interaction, where $r$ runs from 1 up to the coordination number (in our case up to 4 ); $D$ is the single ion-anisotropy parameter acting on spin- $3 / 2$; $S_{i}$ represents the spin-3/2 particle; whereas $\sigma_{i}$ corresponds the spin-1/2 particle, with two possible values \pm 1 (we use these values conveniently instead of $\pm 1 / 2$ for all spin $\sigma_{i}$ ).

First we would like to obtain an exactly solvable spin-(3/2,1/2) lattice $\mathcal{L}_{b}$ (see fig.1); to this end, we write 
the first term of the Hamiltonian (11) as $\left(K_{r}^{(1)} S_{i}+K_{r}^{(2)} S_{i}^{3}\right) \sigma_{j}$, then for spin-3/2 we have four possible values $\pm 1 / 2$ and $\pm 3 / 2$, thus the term $K_{r}^{(1)} S_{i}+K_{r}^{(2)} S_{i}^{3}$ can be written

$$
\begin{aligned}
& A_{r}^{(1)}= \pm \frac{1}{2} K_{r}^{(1)} \pm \frac{1}{8} K_{r}^{(3)}, \quad S_{i}= \pm 1 / 2, \\
& A_{r}^{(2)}= \pm \frac{3}{2} K_{r}^{(1)} \pm \frac{27}{8} K_{r}^{(3)}, \quad S_{i}= \pm 3 / 2,
\end{aligned}
$$

with $r=1, \ldots, 4$.

In order to project the spin-3/2 onto spin $\sigma$ with only two possible values \pm 1 , we impose the condition $\left|A_{r}^{(1)}\right|=\left|A_{r}^{(2)}\right|=A_{r}$. Therefore we are able to find the parameters $K_{r}^{(1)}$ and $K_{r}^{(3)}$ as a function of $A_{r}$, by solving the system (2) and (3), we obtain following

$$
\left\{\begin{array}{l}
K_{r}^{(3)}=-\frac{4}{3} A_{r} ; \quad K_{r}^{(1)}=\frac{7}{3} A_{r} \\
\text { or } \quad, \text { in which } \quad r=1, \ldots, 4 . \\
K_{r}^{(3)}=-\frac{2}{3} A_{r} ; \quad K_{r}^{(1)}=-\frac{13}{6} A_{r}
\end{array}\right.
$$

The associated Boltzmann weight for a mixed spin-(3/2,1/2) lattice has a similar structure to that of the model discussed by $\mathrm{Wu}$ and Lin 24, the associated Boltzmann weights are given by $W\left(\left\{\sigma_{r}\right\}\right)=$ $\sum_{S} \exp \left(\mathcal{H}_{1 / 2,3 / 2}\right)$. For simplicity we consider our calculation in units of $-\beta$. Using the solution given by (4) the associated Boltzmann weights are simplified, which read as

$$
\begin{aligned}
& w_{1}=W(+,+,+,+)=\alpha \cosh \left(A_{1}+A_{2}+A_{3}+A_{4}\right), \\
& w_{2}=W(+,-,+,-)=\alpha \cosh \left(A_{1}-A_{2}+A_{3}-A_{4}\right), \\
& w_{3}=W(+,-,-,+)=\alpha \cosh \left(A_{1}-A_{2}-A_{3}+A_{4}\right), \\
& w_{4}=W(+,+,-,-)=\alpha \cosh \left(A_{1}+A_{2}-A_{3}-A_{4}\right), \\
& w_{5}=W(+,-,+,+)=\alpha \cosh \left(A_{1}-A_{2}+A_{3}+A_{4}\right), \\
& w_{6}=W(+,+,+,-)=\alpha \cosh \left(A_{1}+A_{2}+A_{3}-A_{4}\right), \\
& w_{7}=W(+,+,-,+)=\alpha \cosh \left(A_{1}+A_{2}-A_{3}+A_{4}\right), \\
& w_{8}=W(-,+,+,+)=\alpha \cosh \left(-A_{1}+A_{2}+A_{3}+A_{4}\right),
\end{aligned}
$$


where $\alpha=2\left(\mathrm{e}^{\frac{D}{4}}+\mathrm{e}^{\frac{9 D}{4}}\right)$.

The lattice $\mathcal{L}_{b}$ can be transformed into an effective spin-1/2 lattice $\mathcal{L}_{a}$, as shown in fig.1. Then at each site of $\mathcal{L}_{a}$ there can be eight lines; we are thus led to consider an eight-vertex model $\mathcal{L}_{a}$ with weights given by (5D) and which satisfies the free fermion condition 25,

$$
\omega_{1} \omega_{2}+\omega_{3} \omega_{4}=\omega_{5} \omega_{6}+\omega_{7} \omega_{8}
$$

Consider the following Hamiltonian,

$$
\mathcal{H}_{1 / 2}=J_{0} N+\sum_{(i, j)} J_{i, j} \sigma_{i} \sigma_{j}+\sum_{\begin{array}{c}
\text { all tetra- } \\
\text { hedron }
\end{array}} J_{1,2,3,4} \sigma_{i_{1}} \sigma_{i_{2}} \sigma_{i_{3}} \sigma_{i_{4}}
$$

for the effective spin-1/2 Ising model $\mathcal{L}_{a}$, which is exactly solvable using the eight-vertex model[26]. Here, $(i, j)$ means summation over all pairs of sites over the tetrahedron (the boldface tetrahedron in Fig.1), and $J_{i, j}$ are their corresponding interacting parameters, whereas $J_{1,2,3,4}$ is the quartic interaction parameter; $N$ represents the number of spin-3/2 sites on the lattice.

After transforming the Hamiltonian (1) into (7), we relate their parameters using the Boltzmann weights, thus to obtain

$$
\begin{aligned}
J_{0} & =\ln (2 \alpha)+\frac{1}{8} \ln \left(w_{1} w_{2} w_{3} w_{4} w_{5} w_{6} w_{7} w_{8}\right) \\
J_{1,2} & =\frac{1}{8} \ln \left(\frac{w_{1} w_{4} w_{5} w_{8}}{w_{6} w_{7} w_{2} w_{3}}\right) \\
J_{2,3} & =\frac{1}{8} \ln \left(\frac{w_{1} w_{8} w_{3} w_{6}}{w_{7} w_{4} w_{5} w_{2}}\right) \\
J_{3,4} & =\frac{1}{8} \ln \left(\frac{w_{1} w_{6} w_{7} w_{4}}{w_{5} w_{2} w_{3} w_{8}}\right) \\
J_{4,1} & =\frac{1}{8} \ln \left(\frac{w_{1} w_{7} w_{5} w_{3}}{w_{8} w_{4} w_{2} w_{6}}\right) \\
J_{1,3} & =\frac{1}{8} \ln \left(\frac{w_{1} w_{7} w_{2} w_{8}}{w_{6} w_{4} w_{5} w_{3}}\right) \\
J_{2,4} & =\frac{1}{8} \ln \left(\frac{w_{1} w_{6} w_{5} w_{2}}{w_{7} w_{4} w_{3} w_{8}}\right) \\
J_{1,2,3,4} & =\frac{1}{8} \ln \left(\frac{w_{1} w_{4} w_{2} w_{3}}{w_{8} w_{7} w_{5} w_{6}}\right) .
\end{aligned}
$$

The partition function $\mathcal{Z}$ of the decorated (or mixed) model in the thermodynamic limit is related to the partition function $\mathcal{Z}_{8 v}$ of the effective eight-vertex spin-1/2 model (7) by the expression $\mathcal{Z}=\mathrm{e}^{-\beta J_{0}} \mathcal{Z}_{8 v}$. An 
analytical expression for the free energy of the free fermion model is well known[25] and after some manipulation, was expressed in the thermodynamic limit by

$$
f=J_{0}+\frac{1}{16 \pi^{2}} \int_{0}^{2 \pi} \int_{0}^{2 \pi} \mathrm{d} \theta \mathrm{d} \phi \ln [2 a+2 b \cos (\theta)+2 c \cos (\phi)+2 d \cos (\theta-\phi)+2 e \cos (\theta+\phi)],
$$

where

$$
\begin{aligned}
a & =\frac{1}{2}\left(w_{1}^{2}+w_{2}^{2}+w_{3}^{2}+w_{4}^{2}\right), & b & =w_{1} w_{3}-w_{2} w_{4}, \\
c & =w_{1} w_{4}-w_{2} w_{3}, & d & =w_{3} w_{4}-w_{7} w_{8},
\end{aligned}
$$

The system exhibits an Ising transition at the critical points

$$
w_{1}+w_{2}+w_{3}+w_{4}=2 \max \left\{w_{1}, w_{2}, w_{3}, w_{4}\right\} .
$$

At low temperature the system exhibits ordered states such as the ferromagnetic state (for $w_{1}>w_{2}, w_{3}, w_{4}$ ), the antiferromagnetic state (for $w_{2}>w_{1}, w_{3}, w_{4}$ ) and the metamagnetic state (for $w_{3}$ or $w_{4}>w_{1}, w_{2}$ ).

Thus our goal is to transform an exactly solvable Ising model with spin- $1 / 2$ via the eight-vertex model 26] into an equivalent Ising model on the lattice with spin-3/2. For this purpose we introduce an auxiliary lattice $\mathcal{L}_{b}$, with mixed spin-1/2 and spin-3/2, the schematic transformation of which is displayed in fig 1. If we consider the spin-1/2 model as a decoration of the mixed spin model and transform it into an equivalent spin-3/2 Ising model on lattice, then we can conclude that there exist a transformation from the spin-1/2 Ising model onto the spin-3/2 Ising model, with a non-bilinear interaction and four-body interactions terms over the tetrahedron or quartic interaction.

Using the first of solution of Eq.(4), we express the Hamiltonian of the transformed spin-3/2 lattice $\mathcal{L}_{c}$ in terms of four constrained parameters and one arbitrary parameter $D$, thus obtaining

$$
\begin{aligned}
\mathcal{H}_{3 / 2}= & J_{0} N+\sum_{i} D S_{i}^{2}+\sum_{(i, j)} J_{i, j}\left(\frac{49}{9} S_{i} S_{j}-\frac{28}{9}\left(S_{i} S_{j}^{3}+S_{i}^{3} S_{j}\right)+\frac{16}{9} S_{i}^{3} S_{j}^{3}\right)+\sum_{\substack{\text { all tetra- } \\
\text { hedron }}} J_{1,2,3,4}\left(\frac{2401}{81} S_{i_{1}} S_{i_{2}} S_{i_{3}} S_{i_{4}}\right. \\
& \left.-\frac{1372}{81} S_{i_{1}}^{3} S_{i_{2}} S_{i_{3}} S_{i_{4}}+\frac{784}{81} S_{i_{1}}^{3} S_{i_{2}}^{3} S_{i_{3}} S_{i_{4}}-\frac{448}{81} S_{i_{1}} S_{i_{2}}^{3} S_{i_{3}}^{3} S_{i_{4}}^{3}+\frac{256}{81} S_{i_{1}}^{3} S_{i_{2}}^{3} S_{i_{3}}^{3} S_{i_{4}}^{3}\right),
\end{aligned}
$$

where the parameters $J_{i, j}$ were already defined by Eqs.(8)-(15), and these parameters are constrained. Whereas the parameters $A_{1}, A_{2}, A_{3}$ and $A_{4}$ are free, as well as the single ion anisotropy parameter $D$. Summations are performed as indicated in (7). 
By factorizing the terms under the summation sign, we obtain

$$
\begin{aligned}
\mathcal{H}_{3 / 2}= & J_{0} N+\sum_{i} D S_{i}^{2}+\sum_{(i, j)}\left(J_{i, j} \frac{S_{i} S_{j}}{9}\left(7-4 S_{i}^{2}\right)\left(7-4 S_{j}^{2}\right)\right)+ \\
& +\sum_{\substack{\text { all tetra- } \\
\text { hedron }}} J_{1,2,3,4} \frac{S_{i_{1}} S_{i_{2}} S_{3} S_{i_{4}}}{81}\left(7-4 S_{i_{1}}^{2}\right)\left(7-4 S_{i_{2}}^{2}\right)\left(7-4 S_{i_{3}}^{2}\right)\left(7-4 S_{i_{4}}^{2}\right) .
\end{aligned}
$$

We can reduce the Hamiltonian (20) further for both solutions in Eq.(44), using the spin transformation $\sigma^{(k)}(S)$, where this function only take two possible values \pm 1 , for all values of $S=\{-3 / 2,-1 / 2,1 / 2,3 / 2\}$. These spin transformations read as

$$
\sigma^{(k)}(S)= \begin{cases}\frac{S}{3}\left(7-4 S^{2}\right) ; & k=1 \\ \frac{S}{6}\left(13-4 S^{2}\right) ; & k=2 .\end{cases}
$$

We remark that the transformation given by (21) are the same as those obtained by Izmailian[19] using the method proposed by Wu[18 (but for another model, the spin-3/2 Ising model on a square lattice). As we can see, the previous transformation is independent of the coordination number. Therefore it may be used to yield a particular case of solution, for the exactly solvable spin-3/2 Ising model in a honeycomb lattice as was obtained by Izmailian and Ananikian[20].

We are now able to write the Hamiltonian (20) as

$$
\mathcal{H}_{3 / 2}^{(k)}=J_{0} N+\sum_{i} D S_{i}^{2}+\sum_{(i, j)} J_{i, j} \sigma_{i}^{(k)} \sigma_{j}^{(k)}+\sum_{\begin{array}{c}
\text { all tetra- } \\
\text { hedron }
\end{array}} J_{1,2,3,4} \sigma_{i_{1}}^{(k)} \sigma_{i_{2}}^{(k)} \sigma_{i_{3}}^{(k)} \sigma_{i_{4}}^{(k)} .
$$

The second term of the Hamiltonian (22) may be written using a new spin variable transformation, similar to that performed by Izmailian[19. Note that there the transformation was performed only for spin-3/2 case and for higher spin that method could become a more complex task.

We also remark that the model discussed by Izmailian[19] can be completely re-obtained, by using our simple transformation instead of Wu's 18 method.

The Hamiltonian (10) can be extended to arbitrary half-odd-integer spin-(S,1/2); the general Hamiltonian has the following form

$$
\mathcal{H}_{1 / 2, S}=\sum_{<i, j>}\left(K_{r}^{(1)} S_{i}+K_{r}^{(3)} S_{i}^{3}+K_{r}^{(5)} S_{i}^{5} \cdots+K_{r}^{\left(S+\frac{1}{2}\right)} S_{i}^{S+\frac{1}{2}}\right) \sigma_{j}+\sum_{i} D S_{i}^{2}
$$


where $K_{r}^{(1)}$ is the bilinear interaction parameter, and $K_{r}^{(u)}$ are non-bilinear interaction parameters of $S_{i}^{u} \sigma_{j}$, with $u=2, \ldots, S / 2+1$ and $r=1, \ldots, 4$, thus the set of transformations for each half-odd-integer spin-S have $S+1 / 2$ unknown parameters to be determined. We extend eq.(4) to the general case (23) by constructing a Vandermonde-like matrix, explicitly

$$
\left(\begin{array}{ccccc}
\frac{1}{2} & \left(\frac{1}{2}\right)^{3} & \left(\frac{1}{2}\right)^{5} & \ldots & \left(\frac{1}{2}\right)^{2 S} \\
\frac{3}{2} & \left(\frac{3}{2}\right)^{3} & \left(\frac{3}{2}\right)^{5} & \ldots & \left(\frac{3}{2}\right)^{2 S} \\
\vdots & \vdots & \vdots & \ddots & \vdots \\
S & S^{3} & S^{5} & \ldots & S^{2 S}
\end{array}\right)\left(\begin{array}{c}
K_{r}^{(1)} \\
K_{r}^{(3)} \\
\ldots \\
K_{r}^{\left(S+\frac{1}{2}\right)}
\end{array}\right)=\left(\begin{array}{c}
A_{r}^{(1)} \\
A_{r}^{(2)} \\
\vdots \\
A_{r}^{\left(S+\frac{1}{2}\right)}
\end{array}\right)
$$

where the parameters $A_{r}^{(u)}$ must satisfy the following identities $\left|A_{r}^{(1)}\right|=\left|A_{r}^{(2)}\right|=\cdots=\left|A_{r}^{\left(S+\frac{1}{2}\right)}\right|=A_{r}$, in order to project the spin-S onto spin-1/2. By inverting the Vandermonde-like matrix, we are able to obtain the solution of the algebraic system. In what follows, we show the solutions of these system of linear equations for some higher spin-S values.

For $S=5 / 2$, there are three solutions:

$$
\sigma^{(k)}(S)= \begin{cases}\frac{1067}{480} S-\frac{11}{12} S^{3}+\frac{1}{10} S^{5} ; & k=1 \\ \frac{529}{240} S-\frac{5}{6} S^{3}+\frac{1}{15} S^{5} ; & k=2 \\ \frac{1183}{480} S-\frac{23}{12} S^{3}+\frac{7}{30} S^{5} ; & k=3 .\end{cases}
$$

For $S=7 / 2$, there are four solutions:

$$
\sigma^{(k)}(S)= \begin{cases}\frac{30251}{13440} S-\frac{301}{288} S^{3}+\frac{61}{360} S^{5}-\frac{1}{126} S^{7} ; & k=1 \\ \frac{60577}{26880} S-\frac{3047}{2880} S^{3}+\frac{127}{720} S^{5}-\frac{11}{1260} S^{7} ; & k=2 \\ \frac{14887}{6720} S-\frac{637}{720} S^{3}+\frac{17}{180} S^{5}-\frac{1}{315} S^{7} ; & k=3 \\ \frac{68123}{26880} S-\frac{1289}{576} S^{3}+\frac{293}{720} S^{5}-\frac{5}{252} S^{7} ; & k=4 .\end{cases}
$$


For $S=9 / 2$, we obtain five solutions:

$$
\sigma^{(k)}(S)= \begin{cases}\frac{5851067}{2580480} S-\frac{46573}{41472} S^{3}+\frac{7501}{34560} S^{5}-\frac{97}{6048} S^{7}+\frac{1}{2592} S^{9} ; & k=1 \\ \frac{2924921}{1290240} S-\frac{813413}{725760} S^{3}+\frac{3727}{17280} S^{5}-\frac{239}{15120} S^{7}+\frac{17}{45360} S^{9} ; & k=2 \\ \frac{5868067}{2580480} S-\frac{334907}{290304} S^{3}+\frac{8117}{34560} S^{5}-\frac{113}{6048} S^{7}+\frac{43}{90720} S^{9} ; & k=3 \\ \frac{5725183}{2580480} S-\frac{37337}{41472} S^{3}+\frac{3593}{34560} S^{5}-\frac{29}{6048} S^{7}+\frac{1}{12960} S^{9} ; & k=4 \\ \frac{6651283}{2580480} S-\frac{506017}{207360} S^{3}+\frac{18341}{34560} S^{5}-\frac{1237}{30240} S^{7}+\frac{13}{12960} S^{9} ; & k=5 .\end{cases}
$$

Using the spin transformations given by eqs.(21), (25), (26) and (27), we are able to recover the transformation obtained by Joseph 21] by considering $k=1$, whereas the remaining transformation are new.

In this report we present a transformation which maps a spin-3/2 lattice, with quartic and non-linear interactions terms, onto an effective spin-1/2 Ising model on lattice. First this transformation was carried out using an auxiliary mixed spin-(3/2,1/2) square lattice with only nearest neighbor interaction term, mapped onto an effective spin- $1 / 2$ or spin-3/2 lattice model, depending on which spin is considered to be the decoration spin. Finally, a systematic way of transformation for higher half-odd-integer spin-S is considered, inverting a Vandermonde like matrix, to obtain a families of mapping between spin-S models and spin-1/2 models, using the one-to-one transformation. Therefore we conclude that there exist a spin transformation, which can be applied to lattice models with arbitrary coordination number, including non-exactly solvable half-odd-integer spin-S models. We also recovered some results previously obtained in the literature 19, 20, as a particular case of solution.

O. Rojas. and S.M. de Souza. thanks CNPq and FAPEMIG for partial financial support.

\section{References}

[1] M. Blume, V. J. Emery, R. B. Griffiths, Phys. Rev. A 3, 1071 (1971).

[2] J. Bernasconi and F. Rys, Phys. Rev. B 4, 3045 (1982).

[3] H. W. Capel, Physica 32, 966 (1966); D. Mukamel and M. Blume, Phys. Rev. A 10 61, (1974).

[4] K. G. Chakraborty and T. Morita, Physica A 129, 415 (1985). 
[5] T. Kaneyoshi, Physica A 164, 730 (1976).

[6] S. Krinsky and D. Furman, Phys. Rev. B 11, 2602 (1975); A. N. Berker and M. Wortis, Phys. Rev. 14, 4946 (1976).

[7] D. M. Soul, M. Wortis and D. Stauffer, Phys. Rev. B 9, 4964 (1974)

[8] A. K. Jain and D. P. Landau, Bull. Am. Phys. Soc. 21, 231 (1976).

[9] Onsager, Phys. Rev. 65,117 (1944).

[10] G.F. Newell, Phys. Rev. 79, 876 (1950).

[11] K. Husimi and I. Syozi, Prog. Theor. Phys. 5, 117 (1950).

[12] T. Horiguchi, Phys. Lett. A 113, 425 (1986).

[13] K. Husimi(1) and I. Syozi,Prog. Theor. Phys. 5, 341 (1950).

[14] I. Syozi, Prog. Theor. Phys. 6, 306 (1951).

[15] M. Kolesík and L. Samaj, Int. J. Mod. Phys. B 6, 1529 (1992).

[16] X. D. Mi and Z. R. Yang, J. Phys. A: Math. Gen. 28, 4883 (1995); Phys. Rev E 49, 3636 (1994).

[17] K. Tang, J. Phys. A: Math. Gen. 21, L1097 (1988).

[18] F. Y. Wu, Phys. Lett. A 117, 365 (1986).

[19] N. Sh. Izmailian, Pis'ma Zh. Éksp. Teor. Fiz. 63, 270-275 (1996)

[20] N. Sh. Izmailian and N. S. Ananikian, Phys. Rev. B 50, 6829 (1994).

[21] R. I. Joseph, J. Phys. A:Math. Gen. 9, L31 (1976).

[22] T. Horiguchi, Physica A 214,452 (1995).

[23] J. Strečka, L. Čanová and Ján Dely, Phys. Stat. Solidi (B) 243, 1946 (2006). 
[24] F. Y. Wu and K. Y. Lin, J. Phys. A: Math. Gen. 20 5737, (1987).

[25] C. Fan and F. Y. Wu, Phys. Rev. B 2,723 (1970).

[26] R.J. Baxter, Exacty Solved Models in Statistical Mechanics, Academic Press, N.Y. 1982. 


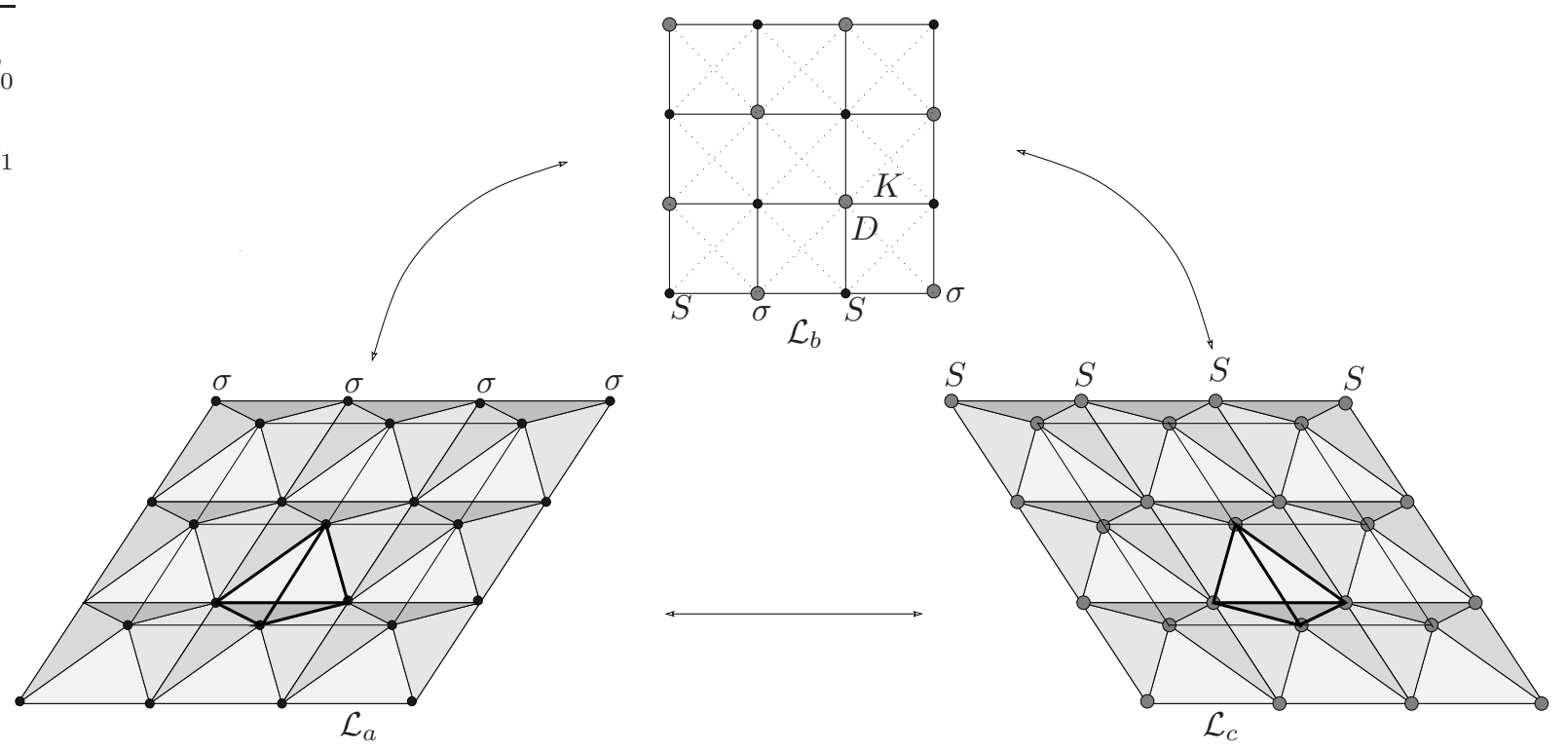

Figure 1: Schematic representation of mixed spin-(S,1/2) on a square lattice $\left(\mathcal{L}_{b}\right)$, a square-type spin-1/2 Ising model $\left(\mathcal{L}_{a}\right)$ and a square-type spin-S Ising model $\left(\mathcal{L}_{c}\right)$ 